\title{
Working with non-medical prescribers
}

As part of the modernisation of the UK healthcare workforce, prescribing authority has been extended to nurses, pharmacists, optometrists, and allied health professionals (AHPs; including radiographers, physiotherapists, podiatrists, and chiropodists). ${ }^{1}$ Although several countries, for example Sweden and the US, have implemented prescribing by non-medical healthcare professionals and it is planned for in others (for example, the Netherlands), no other country has such extended non-medical prescribing (NMP) rights as the UK.

The predicted benefits of the adoption of prescribing by non-medical healthcare professionals include better use of professionals' skills, improved service efficiency and access to medicines, and more flexible team working. ${ }^{1}$ NMP offers an innovative solution to address capacity, quality, efficiency, and effectiveness if used widely in pathway redesign, and should be considered within all service specifications where medicines are prescribed or supplied. ${ }^{1}$

There are now over 50000 NMPs. This includes more than 30000 community practitioners with powers to prescribe from a restricted formulary (mainly overthe-counter medicines and wound dressings), approximately 19000 nurses, nearly 2000 pharmacists, and several hundred AHPs and around 70 optometrists (Department of Health, unpublished data, 2010) with an independent and/or supplementary prescribing qualification. Over 12 million items are prescribed each year by $\mathrm{NMPs}^{2}$ and the extension of prescribing rights to other groups of AHPs (including dieticians) is currently under consideration. $^{3}$

\section{TRAINING NON-MEDICAL PRESCRIBERS}

The NMP programme is shared between nurses, pharmacists, and AHPs (optometrists undergo separate training) and comprises 27 days in the classroom and 12 days supervised practice with a designated medical practitioner (DMP) (Department of Health, unpublished data, 2010). Criteria for applicants to the programme include 3 years' qualified experience (the year immediately preceding application being in the clinical field in which the applicant intends to prescribe), the ability to study at degree level, a DMP who has agreed to provide the required term of supervised practice, the necessary competencies to undertake a history, clinical assessment, and diagnosis, and appropriate numeracy skills (Department of Health, unpublished data, 2010). On completion of the course, nurses and pharmacists are awarded a dual qualification in independent and supplementary prescribing whereas AHPs qualify as supplementary prescribers.

The majority of nurse independent prescribers and nurse supplementary prescribers work in general practice. ${ }^{4}$ Through independent prescribing, these nurses are able to assess, diagnose, and prescribe independently any licensed or unlicensed medicines and some controlled drugs. As supplementary prescribers, they can prescribe any medicine, however, in contrast to independent prescribing, assessment and diagnosis of the patient's condition is carried out by a doctor, and the medicines to be prescribed are detailed in a clinical management plan agreed between the doctor, nurse, and patient (Department of Health, unpublished data, 2010).

\section{PATIENT AND GP PERCEPTIONS}

Patients are confident in nurse prescribing and it is safe. ${ }^{5,6}$ The predicted benefits of the adoption of the prescribing role by nurses have been achieved..$^{5-7}$ In general practice, it is clear that, by developing working relationships with individual nurse prescribers, GPs confidence in nurse prescribing is strengthened. ${ }^{8}$ GPs working alongside nurse prescribers report that they know their nursing colleagues well and are confident in their ability to make prescribing decisions, especially in their management of long- term conditions. ${ }^{8}$ They are confident that nurses are aware of, and work within their competencies referring any uncertain cases to a doctor. They also report that nurse prescribing has 'freed up' their time enabling them to see more complex patients, and learning is increased as a result of greater shared territory and more discussion with nurses about clinical issues. $^{8}$

However, despite the benefits of NMP, its uptake and use is inconsistent ${ }^{9}$ and this is costly in terms of the time and expenses for training individuals to prescribe, and failure to deliver predicted efficiency savings within services. The expansion of prescribing rights in 2006 has been reported to have fuelled fear and misunderstanding by some doctors and that these concerns are more prevalent among medical colleagues without direct experience of this relatively new role for nurses. ${ }^{8}$

\section{PRESCRIBING SAFELY}

While doctors trust the nurse prescribers whom they know, they are fearful that there may be nurses who are prescribing beyond their level of competence and who over prescribe; that is, the 'loose cannon'. ${ }^{8}$ In addition to these beliefs, doctors working with nurse prescribers experience them to have a high level of knowledge, be aware of their personal limitations, and have a cautious attitude. Acceptance by some doctors has been reported to be conditional on the level of regulation or control over what nurses can prescribe, that is, protocols and clinical management plans providing reassurance that they will prescribe safely within their areas and levels of competence.

NMPs can prescribe any medicine from the British National Formulary, although they are careful always to stay within their area of competence, or scope of practice. $^{8}$ If they do not do this, they would bring fitness to practice into question and endanger registration. ${ }^{10}$ While prerequisites for prescribing training include the ability to study at 
degree level and 3 years' qualified experience, evidence suggests that the majority of nurse prescribers have exceeded this level of experience. Most have up to 10 years' experience as a nurse ( 5 of these years being in the area in which they prescribe) and nearly all have academic and specialist qualifications at degree and masters level; 4,5 this provides confidence that selection criteria are in place across organisations.

Reassurance that NMPs will not prescribe outside of their area of competence can also be provided by a 'scope of practice' document. Such a document, in place across many organisations, details the conditions and medicines to be prescribed by the nonmedical healthcare professional, mode of prescribing (that is, independent or supplementary), and evidence of competence and CPD. However, although such documents can be useful, they can limit practice if too stringently defined. A balance is required, whereby prescribing practice is supported without being too restrictive.

\section{CONCLUSION}

Although NMP offers a strategic innovative solution to address capacity, quality, efficiency, and effectiveness, its implementation is inconsistent and concerns among doctors exist. The standards that govern $\mathrm{NMPs}^{10}$ and the available evidence should help to allay some of these anxieties. However, the lack of awareness and understanding by doctors about this literature is concerning, given the new White Paper reforms $^{11}$ and the need for GP-led consortia to include these front-line clinicians in decision making about pathway redesign and care packages.

\section{Molly Courtenay,}

Professor of Clinical Practice, Division of Health and Social Care, Faculty of Health \& Medical Sciences, University of Surrey, Guildford.

\section{Clare Gerada,}

Director, Practitioner Health Programme and Chair, RCGP, London.

\section{Jane Haywood,}

Associate Director, Practitioner Health Programme, Riverside Medical Practice, London.

\section{Provenance}

Commissioned, not externally peer reviewed.

\section{REFERENCES}

1. Department of Health. Improving patients' access to medicines: A guide to implementing nurse and pharmacist independent prescribing within the NHS in England. London: Department of Health, 2006.

2. NHS Prescription Service. Report: update on growth in prescription volume and cost in the year to March 2010. London: NHS Prescription Service, 2010.

3. Department of Health. Allied health professions prescribing and medicines supply mechanisms scoping project report. London: Department of Health, 2010.

4. Courtenay M, Carey N. Nurse independent prescribing and nurse supplementary prescribing practice: national survey. J Adv Nurs 2008, 61(3): 291-299.

5. Drennan J, Naughton C, Allen D, et al. National independent evaluation of the nurse and midwife prescribing initiative. Dublin: University College Dublin, 2009.

6. Courtenay M, Carey N, Stenner K, et al. Patients' views of nurse prescribing: effects on care, concordance and medicine taking. Br J Dermatol 2010, DOI 10.1111/j.1365-2133.2010.10119.x.

7. Courtenay M, Stenner K, Carey N. The views of patients with diabetes about nurse prescribing. Diabet Med 2010; 27(9): 1049-1054.

8. Stenner K, Carey N, Courtenay M. Nurse prescribing in dermatology: doctors' and non-prescribing nurses views. J Adv Nurs 2009; 65(4): 851-859.

9. National Prescribing Centre. Non-medical prescribing by nurses, optometrists, pharmacists, physiotherapists, podiatrists and radiographers: a quick guide for commissioners. Liverpool: National Prescribing Centre, 2010.

10. Nursing and Midwifery Council. Standards of proficiency for nurse and midwife prescribers. London: Nursing and Midwifery Council, 2010.

11. Department of Health. Equity and excellence: liberating the NHS. London: Department of Health, 2010.

DOI: 10.3399/bjgp11X556155

\section{ADDRESS FOR CORRESPONDENCE}

\section{Molly Courtenay,}

Division of Health and Social Care, Faculty of Health \& Medical Sciences, University of Surrey, Guildford GU2 7TE.

E-mail:m.courtenay@surrey.ac.uk 